\title{
Complex Plateau problem in non-Kähler manifolds
}

\author{
by S. IvashKovich (Lille and L'viv)
}

\begin{abstract}
We consider the complex Plateau problem for strongly pseudoconvex contours in non-Kähler manifolds. We give a necessary and sufficient condition for the existence of solution in the class of manifolds carrying pluriclosed metric forms and propose a conjecture for the general case.
\end{abstract}

0. Introduction. Recall that the complex Plateau problem for a compact real submanifold $M$ of a complex manifold $X$ consists in finding an analytic chain $A \subset X \backslash M$ with "boundary" $M$. More precisely, one wants to find a complex analytic subset $A \subset X \backslash M$ such that $\partial[A]=[M]$ in the sense of currents. A necessary condition on $M$ for the complex Plateau problem to have a solution is the maximal complexity of $M$, i.e. $M$ should be a CR-submanifold of $X$ with $\operatorname{dim}_{\mathrm{CR}} M=p$, where $\operatorname{dim}_{\mathbb{R}} M=2 p+1$. In the case when $X$ is Stein this is also sufficient (see $[\mathrm{H}]$ ). Already in the case $X=\mathbb{C P}^{3}$ the maximal complexity of the "contour" $M$ is not sufficient any more $[\mathrm{Db}]$. We refer the interested reader to $[\mathrm{Db}-\mathrm{H}]$ for an extensive exposition on the Plateau problem in projective space.

In this paper we restrict ourselves to strongly pseudoconvex contours $M$, which are already the boundaries of some abstract complex manifolds. At the same time we look for solutions of the complex Plateau problem in more general ambient manifolds.

Let $w$ be a strictly positive, smooth $(1,1)$-form on the complex manifold $X$.

Definition 0.1. We say that $w$ is pluriclosed if $d d^{c} w=0$.

The Hermitian metric canonically associated with such a $w$ is often also called pluriclosed. Denote by $\Delta$ the unit disk in $\mathbb{C}$.

Definition 0.2. We say that a complex space $X$ is disk-convex in dimension $k$ if for any compact set $K \Subset X$ there is another compact set $\widehat{K}$

1991 Mathematics Subject Classification: Primary 32D15.

Key words and phrases: meromorphic map, continuity principle, Hartogs extension theorem, spherical shell, complex Plateau problem. 
such that for every meromorphic mapping $f: \bar{\Delta}^{k} \rightarrow X$ with $f\left(\partial \Delta^{k}\right) \subset K$ one has $f\left(\bar{\Delta}^{k}\right) \subset \widehat{K}$. If $X$ is disk-covex in dimension one, we say simply that $X$ is disk-convex.

All compact spaces are of course disk-convex. More generally, all $k$-convex spaces are disk-convex in dimension $k$.

Definition 0.3. A maximally complex CR-manifold $M$ is called strongly pseudoconvex if it can be realized as a strongly pseudoconvex hypersurface in some complex manifold. We say that $M$ bounds an abstract smooth Stein domain if there exists a CR-imbedding $i: M \rightarrow \Omega$ of $M$ into a complex manifold $\Omega$ such that $i(M)$ bounds in $\Omega$ a Stein domain $D$.

If we say that a CR-manifold $M$ is contained in a complex manifold $X$ we mean that some CR-imbedding $M \rightarrow X$ is given.

TheOREM. Let $M$ be a strongly pseudoconvex, maximally complex, compact CR-manifold in a disk-convex complex manifold $X$ carrying a pluriclosed Hermitian metric form. Suppose that $M$ bounds an abstract smooth Stein domain.

(a) If $\operatorname{dim} M \geq 5$ then the complex Plateau problem for $M \subset X$ has a solution.

(b) If $\operatorname{dim} M=3$ then the complex Plateau problem for $M \subset X$ has a solution iff $M$ is homologous to zero in $X$.

Remarks. 1. Let $H^{2}:=\mathbb{C}^{2} \backslash\{0\} /(z \sim 2 z)$ be the Hopf surface. Take $M$ to be the image of the standard unit sphere from $\mathbb{C}^{2}$ under the natural projection $\pi: \mathbb{C}^{2} \backslash\{0\} \rightarrow H^{2}$. Then $M$ is not homologous to zero in $H^{2}$ (i.e. it is a spherical shell in $H^{2}$ !), so the complex Plateau problem has no solution for this $M$. Note that

$$
w=\frac{i}{2} \frac{d z_{1} \wedge d \bar{z}_{1}+d z_{2} \wedge d \bar{z}_{2}}{\|z\|^{2}}
$$

is a pluriclosed Hermitian metric form on $H^{2}$. Here $\|\cdot\|$ stands for the Euclidean norm in $\mathbb{C}^{2}$.

In the case (a), i.e. when $\operatorname{dim} M \geq 5$ the spherical shells in $X$ are not obstructions to finding a film with boundary $M$ because we have "enough concavity".

2. Consider the Hopf three-fold $H^{3}:=\mathbb{C}^{3} \backslash\{0\} /(z \sim 2 z)$. In this case take a sphere $\mathbb{S}^{3}$ in the hyperplane $\left\{z_{1}=0\right\}$. Its image $M$ under the natural projection will be homologous to zero but will not bound any analytic set in $H^{3}$. The reason here is that $H^{3}$ does not admit a pluriclosed Hermitian metric.

3. If one does not require strict pseudoconvexity of a "contour" $M$ then counterexamples are known already in $\mathbb{C P}^{3}$ (see $[\mathrm{Db}]$ ). 
4. The condition on $M$ to bound an abstract smooth Stein domain is really restrictive in dimension 3, while for higher dimensions one has the Rossi theorem guaranteeing the existence of a (not smooth in general) abstract Stein domain with boundary $M$, (see [Rs]).

5. When $X$ is Kähler then any strongly pseudoconvex $M \subset X$ which bounds an abstract Stein domain is homologous to zero in $X$. This follows from the Hartogs-type extension theorem proved in [Iv-1].

The proof of the Theorem consists in meromorphic extension of a CRimbedding of $M$ into $X$ onto the Stein domain bounded by this $M$. We do it along the levels of an appropriate plurisubharmonic Morse exhaustion function (see $\S \S 2$ and 3 ).

In the last $\S 4$ we give some open questions.

1. Continuity principle and spherical shells. To realize this approach we need some results on extension of meromorphic mappings into general complex manifolds (spaces). For the convenience of the reader we collect them in this section.

We denote by $|\cdot|$ the polydisk norm in $\mathbb{C}^{n}$. Put $A^{k}(r, 1):=\left\{z \in \mathbb{C}^{k}\right.$ : $r<|z|<1\}$ and $A_{s}^{k}(r, 1):=\{s\} \times A^{k}(r, 1)$ for $s \in \Delta^{n}$. Let $f: \Delta^{n} \times$ $A^{k}(r, 1) \rightarrow X$ be a holomorphic mapping into a normal complex space $X$. Denote by $S$ the set of points $s \in \Delta^{n}$ such that the restriction $f_{s}:=\left.f\right|_{A_{s}^{k}(r, 1)}$ extends meromorphically onto the polydisk $\Delta_{s}^{k}:=\{s\} \times \Delta^{k}$.

We say that a set $S \subset \Delta^{n}$ is thick at the point $z_{0}$ if for any neighborhood $U \ni z_{0}, S \cap U$ is not contained in a proper analytic subset of $U$.

Theorem 1.1 (Continuity principle). Let $f: \Delta^{n} \times A^{k}(r, 1) \rightarrow X$ be a holomorphic mapping into a normal complex space $X$, disk-convex in dimension $k$. Suppose that there is a constant $C_{0}<\infty$ and a compact set $K \subset X$ such that for $s$ in some subset $S \subset \Delta^{n}$, which is thick at the origin:

(a) the restriction $f_{s}:=\left.f\right|_{A_{s}^{k}(r, 1)}$ is well defined and extends meromorphically onto the polydisk $\Delta_{s}^{k}=\{s\} \times \Delta^{k}$, and $\operatorname{vol}\left(\Gamma_{f_{s}}\right) \leq C_{0}$ for all $s \in S$;

(b) $f\left(\Delta^{n} \times A^{k}(r, 1)\right) \subset K$ and $f_{s}\left(\Delta^{k}\right) \subset K$ for all $s \in S$.

Then:

(1) If $n=1$ then there is a neighborhood $U \ni 0$ in $\Delta$ such that $f$ extends meromorphically onto $U \times \Delta^{k}$.

(2) If $n \geq 2$ and $X$ has bounded cycle geometry in dimension $k$, then again there is a neighborhood $U \ni 0$ in $\Delta^{n}$ and a meromorphic extension of $f$ onto $U \times \Delta^{k}$.

Here $\Gamma_{f_{s}}$ denotes the graph of $f_{s}$ and the volumes are taken with respect to some Hermitian metric $h$ on $X$ and the standard Euclidean metric on $\mathbb{C}^{k}$. 
The condition of finiteness clearly does not depend on the particular choice of the metric.

Let us explain the boundary cycle geometry condition in (2). Denote by $\mathcal{B}_{k}(X)$ the Barlet space of compact analytic cycles of dimension $k$ in $X$, i.e. finite sums $\sum_{j} n_{j} Z_{j}$, where $n_{j} \in \mathbb{N}$ and $Z_{j}$ are $k$-dimensional, compact, irreducible analytic subsets of $X$. One equips $\mathcal{B}_{k}(X)$ with the usual cycle topology (or equivalently with the locally flat topology on currents of integration over those analytic subsets). $\mathcal{B}_{k}(X)$ admits the structure of a finite-dimensional analytic space, compatible with this topology (see [Ba]). This space has at most a countable number of irreducible components.

Definition 1.1. We say that a complex space $X$ has bounded cycle geometry in dimension $k$ if all connected components of the Barlet space $\mathcal{B}_{k}(X)$ are compact.

In other words, $X$ has bounded cycle geometry in dimension $k$ if all irreducible components of $\mathcal{B}_{k}(X)$ are compact and all connected components of $\mathcal{B}_{k}(X)$ are just finite unions of irreducible ones. Note again that the property of having bounded cycle geometry does not depend on the choice of a Hermitian metric.

For the proof of this theorem we refer to [Iv-2]. There also an example is given, showing that in the case $n \geq 2$ some condition on $X$ is needed, in contrast to the case $n=1$.

There are some cases, occurring in applications, when the condition of bounded cycle geometry can be dropped. For example one has the following

Proposition 1.2. Let $f: \Delta^{n} \times A^{k}(r, 1) \rightarrow X$ be a holomorphic map into a normal complex space $X$, disk-convex in dimension $k$. Suppose that:

(1) for every $s \in \Delta^{n}$ the restriction $f_{s}$ extends meromorphically onto $\Delta_{s}^{k}$;

(2) there is a compact $K \Subset X$ such that $f_{s}\left(\Delta_{s}^{k}\right) \subset K$ for all $s$ and $f\left(\Delta^{n} \times A^{k}(r, 1)\right) \Subset K$

(3) the volumes of the graphs $\Gamma_{f_{s}}$ are uniformly bounded in $\Delta^{n}$, i.e. there exists $C_{0}<\infty$ such that $\operatorname{vol}\left(\Gamma_{f_{s}}\right) \leq C_{0}$ for all $s$.

Then $f$ meromorphically extends onto $\Delta^{n+k}$.

For the proof see [Iv-2], Corollary 1.

It is probably worth to point out one case when the boundedness of cycle geometry is satisfied automatically: when $k=\operatorname{dim} X-1$. Really the cycle space of divisors is allways compact (provided $X$ is compact).

Denote by

$$
\begin{aligned}
H_{n}^{k}(r) & :=\left\{\left(z^{\prime}, z^{\prime \prime}\right) \in \Delta^{n+k}: 1-r<\left|z^{\prime \prime}\right|<1 \text { or }\left|z^{\prime}\right|<r\right\} \\
& =\Delta^{n} \times A^{k}(1-r, 1) \cup \Delta^{n}(r) \times \Delta^{k}
\end{aligned}
$$

the $k$-concave Hartogs figure in $\mathbb{C}^{n+k}$. 
DeFinition 1.2. We say that the meromorphic mappings into the space $X$ have the Hartogs-type extension property in bidimension $(n, k)$ if any meromorphic map $f: H_{n}^{k}(r) \rightarrow X$ extends meromorphically onto $\Delta^{n+k}$.

Definition 1.3. Let us call a Hermitian form $w$ on $X$ plurinegative if $d d^{c} w \leq 0$.

The class of normal complex spaces admitting a plurinegative Hermitian metric form is denoted by $\mathcal{P}_{-}$. Recall that a subset $A \subset \mathbb{C}^{n}$ is called $p$-polar if for any point $a \in A$ one can find local cooordinates $z_{1}, \ldots, z_{n}$ in the neighborhood of $a$ such that for every $\left(z_{1}^{0}, \ldots, z_{p}^{0}\right)$ with $\left|z_{i}^{0}\right|<1$ the set $A \cap\left\{z_{i}=z_{i}^{0}, i=1, \ldots, p\right\}$ is pluripolar in $\mathbb{C}^{n-p}$.

TheOREM 1.3. Let $f: H_{n}^{1}(r) \rightarrow X$ be a meromorphic map into a disk-convex complex space $X$ which admits a plurinegative Hermitian metric form. Then:

(1) $f$ extends to a meromorphic map $\widehat{f}: \Delta^{n+1} \backslash A \rightarrow X$, where $A$ is a closed $(n-1)$-polar subset of $\Delta^{n+1}$.

(2) If , moreover, $w$ is pluriclosed then $A$ is an analytic subvariety of $\Delta^{n+1}$ of pure codimension two (maybe empty). If $A \neq \emptyset$ then for every sphere $\mathbb{S}^{3}$ embedded in $\Delta^{n+1} \backslash A$ in such a way that $\left[\mathbb{S}^{3}\right] \neq 0$ in $H_{3}\left(\Delta^{n+1} \backslash A, \mathbb{Z}\right)$, also its image $f\left(\mathbb{S}^{3}\right)$ is not homologous to zero in $X$.

REMARKs. 1. One can estimate the number of irreducible components of the singularity set $A$ in this theorem meeting a compact subset $P \Subset \Delta^{n+1}$. Namely, let a compact set $K \subset X$, which contains $\overline{f(P \backslash S)}$, be chosen to be a finite subcomplex of a CW-complex $X$. Choose a point $z^{\prime} \in \Delta^{n-1}$ such that $A$ intersects $\Delta_{z}^{2}:=\{z\} \times \Delta^{2}$ in a discrete set $A_{z^{\prime}}$. Suppose $A_{z^{\prime}} \cap \partial P=\emptyset$. Then

$$
\begin{aligned}
& \left|A_{z^{\prime}} \cap P\right| \\
& \quad \leq\left|\int_{\partial\left(P \cap \Delta_{z^{\prime}}^{2}\right)} d^{c} w\right| \cdot\left[\inf \left\{\left|\int_{\gamma} d^{c} w\right|: \gamma \in H_{3}(K, \mathbb{Z}), \int_{\gamma} d^{c} w \neq 0\right\}\right]^{-1} .
\end{aligned}
$$

In other words, the number of branches of the singular set (and moreover, their existence) is bounded by the differential geometry of $X$. Note that the subset $\left\{\left|\int_{\gamma} d^{c} w\right|: \gamma \in H_{3}(K, \mathbb{Z}), \int_{\gamma} d^{c} w \neq 0\right\} \subset \mathbb{R}$ is separated from zero (see (2.2.14) in [Iv-2]).

2. Let a spherical shell of dimension $k+1$ in a complex space $X$ be the image $\Sigma$ of the standard sphere $\mathbb{S}^{2 k+1} \subset \mathbb{C}^{k+1}$ under a meromorphic map of some neighborhood of $\mathbb{S}^{2 k+1}$ into $X$, such that $\Sigma$ is not homologous to zero in $X$. This notion is close to the notion of the global spherical shell, introduced by Kato (see [Ka-3]). Thus we obtain the following 
COROLlary 1.4. Let $X$ be a disk-convex complex space which has a pluriclosed Hermitian metric form. Then the following are equivalent:

(a) $X$ has the meromorphic extension property in bidimension $(n, 1)$ for all $n \geq 1$, and thus in all bidimensions $(n, k)$.

(b) X contains no two-dimensional spherical shells.

3. A wide class of complex manifolds without two-dimensional spherical shells is for example the class of manifolds $X$ for which the Hurewicz homomorphism $\pi_{3}(X) \rightarrow H_{3}(X, \mathbb{Z})$ vanishes.

We now need to state a lemma which is one of the key points in the proof of the results listed in this section, and which we shall use in the solution of the complex Plateau problem.

Recall first a few well known facts from pluripotential theory. Let $D$ be an open subset of $\mathbb{C}^{n}$ and $S$ a subset of $D$. Consider the class of functions

$$
\mathcal{U}(S, D):=\left\{u \in \mathcal{P}_{-}(D):\left.u\right|_{S} \geq 1\right\}
$$

where $\mathcal{P}_{-}(D)$ denotes the class of nonnegative plurisuperharmonic functions in $D$.

Definition 1.4. The lower regularization $w_{*}$ of the function

$$
w(\zeta, S, D):=\inf \{u(\zeta): u \in \mathcal{U}(S, D)\}
$$

is called the $\mathcal{P}$-measure of $S$ in $D$, i.e.

$$
w_{*}(z, S, D):=\liminf _{\zeta \rightarrow z} w(\zeta, S, D) .
$$

Note that $w_{*}$ is plurisuperharmonic in $D$.

Definition 1.5. A point $s_{0} \in S$ is called a locally regular point of $S$ if $w_{*}\left(s_{0}, S \cap \Delta\left(s_{0}, \varepsilon\right), \Delta\left(s_{0}, \varepsilon\right)\right)=1$ for all $\varepsilon>0$.

We also say that the set $S$ is locally regular at $s_{0}$.

Consider now a meromorphic mapping $f: \Delta^{p} \times \Delta^{q}(a) \rightarrow X$ into a complex space $X$. Here $\Delta^{q}(a)$ is a polydisk in $\mathbb{C}^{q}$ of radii $a, \Delta^{q}(1)=\Delta^{q}$. Let $S$ be some closed subset of $\Delta^{p}$ and $s_{0} \in S$. Suppose that for each $s \in S$ the restriction $f_{s}:=\left.f\right|_{\{s\} \times \Delta^{q}(a)}$ is well defined and meromorphically extends onto a $q$-disk $\Delta^{q}(b), b>a$. Denote by $\nu_{j}=\nu_{j}(K)$ the minimum of the volumes of $j$-dimensional compact analytic subsets contained in some compact set $K \subset X$ (see Lemma 2.3.1 from [Iv-3]). Fix some $a<c<b$. Put

$$
\nu=\min \left\{\operatorname{vol}\left(A_{q-j}\right) \nu_{j}: j=1, \ldots, q\right\},
$$

where $A_{q-j}$ runs over all $(q-j)$-dimensional analytic subsets of $\Delta^{q}(b)$ intersecting $\Delta^{q}(c)$. 
Lemma 1.5. Suppose that there exists a neighborhood $U \ni s_{0}$ in $\Delta^{p}$ such that for all $s_{1}, s_{2} \in S \cap U$,

$$
\left|\operatorname{vol}\left(\Gamma_{f_{s_{1}}}\right)-\operatorname{vol}\left(\Gamma_{f_{s_{2}}}\right)\right|<\nu / 2,
$$

and that $s_{0}$ is a locally regular point of $S$. Then there exists a neighborhood $V_{c} \ni s_{0}$ in $\Delta^{p}$ such that $f$ meromorphically extends onto $V_{c} \times \Delta^{q}(c)$.

For the proof see [Iv-3], Lemma 2.4.1.

2. Three-dimensional contours. In this section we prove part (b) of our Theorem from the Introduction.

We suppose that $M$ bounds an abstract smooth Stein domain, i.e. there is a complex manifold $D$ with boundary $M$ such that $D \backslash M$ is Stein, and that a CR-imbedding $f: M \rightarrow X$ is given. All that we need to prove is that $f$ extends meromorphically onto $D$. Clearly we can suppose that $f$ is already holomorphically extended to some neighborhood of $M$ in $D$.

Proposition 2.1. Let $(D, M)$ be as above and suppose additionally that $\operatorname{dim} M=3$.

(a) Any CR-map $f: M \rightarrow X$, where $X$ is a disk-convex complex space admitting a pluriclosed Hermitian metric form, extends meromorphically onto $D \backslash S$ where $S$ is a finite subset of $D$.

(b) If $f(M)$ is homologous to zero in $X$, or if $X$ does not contain spherical shells, then $S$ is empty.

Pro of. Let $\varrho: D \rightarrow[0,1]$ be a strictly plurisubharmonic Morse exhausting function (see [H-L], p. 93). Define $D_{\varepsilon}^{+}=\{z \in D: \varrho(z)>\varepsilon\}$. Let $\mathcal{E}$ be the set of $\varepsilon$ such that $f$ can be meromorphically extended onto $D_{\varepsilon}^{*} \backslash S_{\varepsilon}$, where $S_{\varepsilon}$ is a discrete set. $\mathcal{E}$ is obviously closed and nonempty. All we need to prove is that $\mathcal{E}$ is open.

Let $\varepsilon_{0}=\inf \{\varepsilon \in \mathcal{E}\}$. If $\varepsilon_{0}$ is a regular value of $\varrho$ then the needed result immediately follows from Theorem 1.3(2).

Consider the case of $\varepsilon_{0}$ not being a regular value of $\varrho$.

Lemma 2.2 below will be proved for arbitrary dimension $n \geq 2$ and used also in $\S 3$.

Denote by $M_{\varepsilon_{0}}=\left\{z: \varrho(z)=\varepsilon_{0}\right\}$ the critical level set. Fix a critical point $z_{0} \in M_{\varepsilon_{0}}$. All we need to prove is that for any neighborhood $W$ of $z_{0}$ the envelope of holomorphy of $W \cap D_{\varepsilon}^{+}$contains some neighborhood of $z_{0}$. For convenience we can suppose that $z_{0}=0$ and $\varepsilon_{0}=0$. Write

$$
\varrho(z)=Q(z)+\langle z, z\rangle+\bar{Q}(z)+O\left(\|z\|^{3}\right),
$$

where $Q(z)$ is a holomorphic polynomial and $\langle z, z\rangle$ the Levi Hermitian form of $\varrho$. By a linear coordinate change we transform $\langle$,$\rangle to the sum of squares$ of absolute values. Then by a unitary coordinate change we transform $Q$ 
to the sum of squares with real nonnegative coefficients. Now (2.1) has the form

$$
\varrho(z)=\sum_{j=1}^{p} a_{j} z_{j}^{2}+\sum_{j=1}^{p} a_{j} \bar{z}_{j}^{2}+\sum_{j=1}^{n}\left|z_{j}\right|^{2}+O\left(\|z\|^{3}\right) .
$$

In coordinates $z_{j}=x_{j}+i y_{j}$ we rewrite (2.2) as follows:

$$
\begin{aligned}
\varrho(z) & =2 \sum_{j=1}^{p} a_{j}\left(x_{j}^{2}-y_{j}^{2}\right)+\sum_{j=1}^{n}\left(x_{j}^{2}+y_{j}^{2}\right)+O\left(\|z\|^{3}\right) \\
& =\sum_{j=1}^{p}\left[\left(1+2 a_{j}\right) x_{j}^{2}+\left(1-2 a_{j}\right) y_{j}^{2}\right]+\sum_{j=p+1}^{n}\left(x_{j}^{2}+y_{j}^{2}\right)+O\left(\|z\|^{3}\right) .
\end{aligned}
$$

Renumber the coordinates in such a way that $a_{j} \geq 1 / 2$ for $j=1, \ldots, q$ and $a_{j}<1 / 2$ for $j=q+1, \ldots, p$. Then

$$
\begin{aligned}
\varrho(z) & \geq \sum_{j=1}^{q}\left[\left(2 a_{j}+1\right) x_{j}^{2}-\left(2 a_{j}-1\right) y_{j}^{2}\right]+\delta \sum_{j=q+1}^{p}\left|z_{j}\right|^{2}+O\left(\|z\|^{3}\right) \\
& \geq \sum_{j=1}^{q}\left[\left(2 a_{j}-\delta_{1}+1\right) x_{j}^{2}-\left(2 a_{j}+\delta_{1}-1\right) y_{j}^{2}\right]+\delta \sum_{j=q+1}^{p}\left|z_{j}\right|^{2}=: \varrho_{1}(z),
\end{aligned}
$$

for some $\delta>0$ and $\delta_{1}$ can be chosen arbitrarily small for small $\|z\|$. As obviously $D^{+}:=\left\{z \in \mathbb{B}^{n}: \varrho_{1}(z)>0\right\} \subset D_{\varepsilon_{0}}^{+}$, all we need to prove is the following

LEMmA 2.2. The envelope of holomorphy of $D^{+}$contains the origin.

Pro of. Consider two cases.

Case 1: $q \leq n-1$. In this case $D^{+}$contains the following "Hartogs figure":

$$
H:=\left\{z \in \mathbb{B}^{n}: \sum_{j=1}^{q}\left[\left(2 a_{j}-\delta_{1}+1\right) x_{j}^{2}-\left(2 a_{j}+\delta_{1}-1\right) y_{j}^{2}\right]>0, \delta \sum_{j=q+1}^{n}\left|z_{j}\right|^{2}<1\right\}
$$

or

$H:=\left\{z \in \mathbb{B}^{n}: \sum_{j=1}^{q}\left[\left(2 a_{j}-\delta_{1}+1\right) x_{j}^{2}-\left(2 a_{j}+\delta_{1}-1\right) y_{j}^{2}\right]>-\varepsilon, \delta \sum_{j=q+1}^{n}\left|z_{j}\right|^{2}>\varepsilon\right\}$.

The envelope of holomorphy of $H$ obviously contains the origin.

CASE 2: $q=n$. In this case

$$
D^{+}=\left\{z \in \mathbb{B}^{n}: \sum_{j=1}^{n}\left[\left(2 a_{j}-\delta_{1}+1\right) x_{j}^{2}-\left(2 a_{j}+\delta_{1}-1\right) y_{j}^{2}\right]>0\right\} .
$$


Put $b_{j}:=2 a_{j}-\delta_{1}+1, c_{j}:=2 a_{j}+\delta_{1}-1, j=1, \ldots, n$. For small $\delta_{1}, b_{j}>c_{j}$. Write (2.5) in the form

$$
D^{+}=\left\{z \in \mathbb{B}^{n}: \sum_{j=1}^{n} b_{j} x_{j}^{2}>\sum_{j=1}^{n} c_{j} y_{j}^{2}\right\} .
$$

In the new coordinates $z_{j} \rightarrow \sqrt{b_{j}} z_{j}$, (2.6) takes the form

$$
D^{+}=\left\{z \in \mathbb{B}^{n}: \sum_{j=1}^{n} x_{j}^{2}>\sum_{j=1}^{n} \delta_{j} y_{j}^{2}\right\},
$$

where $\delta_{j}:=c_{j} / b_{j}<1, j=1, \ldots, n$. Put $\delta_{0}:=\max \left\{\delta_{1}, \ldots, \delta_{n}\right\}<1$. Then

$$
D^{+} \supset D_{1}^{+}=\left\{z \in \mathbb{B}^{n}:\|x\|^{2}>\delta_{0}\|y\|^{2}\right\} .
$$

The set $D_{1}^{+}$clearly contains the following complete "tube torus":

$$
T:=\left\{x+i y \in \mathbb{C}^{n}:\|x\|=1,\|y\| \leq 1 / \delta_{0}\right\},
$$

where $1 / \delta_{0}=: \eta>1$.

We now prove that already the envelope of holomorphy of $T$ contains the origin. For this consider the following continuous family of complex hypersurfaces:

$$
C_{t}=\left\{z \in \mathbb{C}^{n}: z_{1}^{2}+\ldots+z_{n}^{2}=t\right\}
$$

$$
C_{t}=\left\{x+i y \in \mathbb{C}^{n}:\|x\|^{2}-\|y\|^{2}=t, \quad(x, y)=0\right\},
$$

where $(x, y)=x_{1} y_{1}+\ldots+x_{n} y_{n}$. Consider the intersection of $C_{t}$ with the ball of radius $2-t$ :

$$
\widetilde{C}_{t}=\left\{x+i y \in \mathbb{B}_{2-t}^{n}:\|x\|-\|y\|=t,(x, y)=0\right\} .
$$

This is a continuous family of irreducible analytic hypersurfaces with boundaries such that

$$
\begin{aligned}
\partial \widetilde{C}_{t} & =\left\{x+i y \in \partial \mathbb{B}_{2-t}^{n}:\|x\|^{2}-\|y\|^{2}=t,(x, y)=0\right\} \\
& =\left\{x+i y:\|x\|^{2}+\|y\|^{2}=2-t,\|x\|^{2}-\|y\|^{2}=t,(x, y)=0\right\} \\
& =\left\{x+i y:\|x\|^{2}=1,\|y\|^{2}=1-t\right\} \subset T .
\end{aligned}
$$

But $\widetilde{C}_{0} \ni 0$ and $\widetilde{C}_{1} \subset T$. So, by the continuity principle the envelope of holomorphy of $T$ contains the origin.

End of proof. So, as in the case of a regular value, we can extend our map $f$ meromorphically to a neighborhood of the critical level $M_{\varepsilon_{0}}$ minus a discrete set. As a result we obtain an extension $\widehat{f}$ of our map onto $\bar{D} \backslash S$ where $S$ is a finite subset of $\bar{D}$ not intersecting $M=\partial D$. If we put $T:=f^{*} w$ then $d d^{c} \widetilde{T}$ is a nonpositive measure supported on $S$ (see Lemma 2.6.1 from 
[Iv-2]). We have

$$
\int_{S} d d^{c} \widetilde{T}=\int_{D} d d^{c} \widetilde{T}=\int_{\partial D} d^{c} T=\int_{f(\partial D)} d^{c} w=\int_{M} d^{c} w=0
$$

if $M$ is homologous to zero in $X$, or if $X$ does not contain spherical shells. Part (b) of the Theorem is proved.

3. Higher-dimensional contours. We now prove part (a) of our Theorem. We need the following statement. Let $S$ be a closed subset in the product $W=B^{n-1} \times B^{2}$ of two balls such that $S \cap\left(\overline{B^{n-1}} \times \partial B^{2}\right)=\emptyset$. For $z^{\prime} \in B^{n-1}$ set $B_{z^{\prime}}^{2}:=\left\{z^{\prime}\right\} \times B^{2}$. Let a meromorphic mapping $f: W \backslash S \rightarrow X$ be given, where $X$ carries a pluriclosed metric form $w$. Suppose that $f$ is holomorphic in a neighborhood of $\overline{B^{n-1}} \times \partial B^{2}$. Denote by $T$ the preimage of $w$ by $f$ and let $\widetilde{T}$ be the trivial extension of $T$ onto $W$. We suppose here that $\widetilde{T}$ exists (see $[\mathrm{Lg}]$ ).

Lemma 3.1. Suppose that for all $z^{\prime} \in B^{n-1}, f\left(B_{z^{\prime}}^{2}\right)$ is homologous to zero in $X$. Then:

(i) $d d^{c} \widetilde{T}=0$ in the sense of currents.

(ii) There is a (1,0)-current $\gamma$ on $W$, smooth in the neighborhood of $\overline{B^{n-1}} \times \partial B^{2}$, such that $\widetilde{T}=i(\partial \bar{\gamma}-\bar{\partial} \gamma)$.

For the proof see [Iv-2], Lemma 2.6.2.

Note that only at the end of the proof in $\S 2$ did we use the fact that the dimension of $D$ is two. So the following proposition clearly enables us to finish the proof also of part (a) of the Theorem, i.e. for $\operatorname{dim} D \geq 3$.

Proposition 3.2. Every holomorphic map $f$ from $H_{1}^{n}(r)$ into a diskconvex complex space $X$ which admits a pluriclosed Hermitian metric extends meromorphically onto $\Delta^{n}$ provided $n \geq 2$.

Proof. It will be convenient for us to prove simultaneously the main statement of the proposition, and the following weaker statement. Define $A^{n}(a, b):=\Delta^{n}(b) \backslash \Delta^{n}(a)$, for $0 \leq a<b$.

Every holomorphic map $f: A^{n}(1 / 2,1) \rightarrow X$, where $X$ is from Proposition 3.2 , extends meromorphically onto $\Delta^{n}$, provided $n \geq 2$ and $f\left(\partial \Delta_{3 / 4}^{n}\right)$ is homologous to zero in $X$.

We prove both statements by induction on $n$. For $n=2$ the second statement follows directly from Theorem 1.3. So it is sufficient to prove that for any $n \geq 2$ the second statement implies the statement of the proposition for this $n$.

So let a holomorphic mapping $f: H_{1}^{n}(r) \rightarrow X$ be given. For every $z \in \Delta$ the restriction $f_{z}$ of $f$ to $\Delta_{z}^{n}:=\{z\} \times \Delta^{n}$ is holomorphic on $A^{n}(r, 1)$. So, by the assumption $f_{z}$ meromorphically extends onto $\Delta^{n}$, be- 
cause $f\left(\partial \Delta_{z}^{n}\right) \sim f\left(\partial \Delta_{0}^{n}\right) \sim 0$ in $X$ ! Lemma 1.5 immediately gives us (after shrinking $\Delta^{n+1}$ and taking different slopes of $z_{2}, \ldots, z_{n+1}$-direction) the meromorphic extension of $f$ onto $\Delta^{n+1} \backslash S$ where $S$ is a zero-dimensional pluripolar compact set in $\Delta^{n+1}$.

Because $I(f)$ is an analytic set a positive dimension outside a zerodimensional set, $\overline{I(f)}$ is analytic in $\Delta^{n+1} \backslash H_{1}^{n}$, and thus empty. So the fundamental set of $f$ is discrete in $\Delta^{n+1} \backslash S$.

Put $T:=f^{*} w$, where $w$ is a pluriclosed metric form on $X$. Then $T$ has locally summable coefficients in $\Delta^{n+1}$ and its trivial extension $\widetilde{T}$ is plurinegative with $d d^{c} \widetilde{T}$ supported on $S$. Observe that $\widetilde{T}=T$ is pluriclosed outside $S$.

Lemma 3.1 tells us that $d d^{c} \widetilde{T}=0$ and moreover there is a $(1,0)$-current $\gamma$ in any given ball $W \subset \Delta^{n+1}, \partial W \cap S=\emptyset$, smooth on $W \backslash S$, such that $\widetilde{T}=i(\partial \bar{\gamma}-\bar{\partial} \gamma)$. Note that the conditions of Lemma 3.1 are satisfied, because $S$ is zero-dimensional and $n+1 \geq 3$. It remains to repeat the arguments from the proof of Lemma 2.6.3 of [Iv-2] to estimate the volume of the graph of $f$ in the neighborhood of $S$. Namely,

$$
\begin{aligned}
\operatorname{Vol} & \left(\Gamma_{\left.f\right|_{W \backslash S}}\right) \\
& =\int_{W \backslash S}\left(T+d d^{c}\|z\|^{2}\right)^{n+1}=\sum_{j=0}^{n+1} C_{n+1}^{j} \int_{W \backslash S} T^{j} \wedge\left(d d^{c}\left\|z^{\prime}\right\|^{2}\right)^{n+1-j} \\
& \leq C \int_{W \backslash S} T^{j} \wedge\left(d d^{c}\left\|z^{\prime}\right\|^{2}\right)^{n+1-j}=C \lim _{\varepsilon \backslash 0} \int_{W \backslash S} \widetilde{T}_{\varepsilon}^{j} \wedge\left(d d^{c}\left\|z^{\prime}\right\|^{2}\right)^{n+1-j} \\
& \leq C \lim _{\varepsilon \backslash 0} \int_{W} \widetilde{T}_{\varepsilon}^{j} \wedge\left(d d^{c}\left\|z^{\prime}\right\|^{2}\right)^{n+1-j} \\
& =C \lim _{\varepsilon \searrow 0} \int_{W}\left(\partial \bar{\gamma}_{\varepsilon}^{1,0}+\bar{\partial} \gamma_{\varepsilon}^{1,0}\right)^{j} \wedge\left(d d^{c}\left\|z^{\prime}\right\|^{2}\right)^{n+1-j} \\
& =C \lim _{\varepsilon \searrow 0} \int_{W}\left(d\left(\bar{\gamma}_{\varepsilon}^{1,0}+\gamma_{\varepsilon}^{1,0}\right)\right)^{j} \wedge\left(d d^{c}\left\|z^{\prime}\right\|^{2}\right)^{n+1-j} \\
& =C \lim _{\varepsilon \backslash 0} \int_{\partial W}\left(\bar{\gamma}_{\varepsilon}^{1,0}+\gamma_{\varepsilon}^{1,0}\right) \wedge d\left(\bar{\gamma}_{\varepsilon}^{1,0}+\gamma_{\varepsilon}^{1,0}\right)^{j-1} \wedge\left(d d^{c}\left\|z^{\prime}\right\|^{2}\right)^{n+1-j} \\
& =C \int_{\partial W}\left(\bar{\gamma}^{1,0}+\gamma^{1,0}\right) \wedge d\left(\bar{\gamma}^{1,0}+\gamma^{1,0}\right)^{j-1} \wedge\left(d d^{c}\left\|z^{\prime}\right\|^{2}\right)^{n+1-j}<\infty .
\end{aligned}
$$

From the Bishop theorem we get an extension of the graph of $f$ onto $\Delta^{n+1}$.

4. Open questions. Let us propose some open questions arising naturally from the exposition. Our point of departure will be the following observation due to Gauduchon: 
Every compact complex manifold of dimension $k+1$ carries a Hermitian metric form $w$ with $d d^{c} w^{k}=0$.

Really, the condition to carry a $d d^{c}$-closed strictly positive $(k, k)$-form for a compact complex manifold is equivalent to that of carrying a bidimension $(k+1, k+1)$ current $T$ with $d d^{c} T \geq 0$ but $\not \equiv 0$. In the case of $\operatorname{dim} X=k+1$ this is a nonconstant plurisubharmonic function, which does not exist on $X$ compact. In fact in $[\mathrm{Ga}]$ a stronger statement was proved, but we shall not need it here.

Let us introduce the class $\mathcal{G}_{k}$ of normal complex spaces carrying a nondegenerate positive $d d^{c}$-closed strictly positive $(k, k)$-form. Note that the sequence $\left\{\mathcal{G}_{k}\right\}$ is rather exhaustive: $\mathcal{G}_{k}$ contains all compact complex manifolds of dimension $k+1$.

Note also that compact spaces from $\mathcal{G}_{k}$ have bounded cycle geometry in dimension $k$ (see 1.4 from [Iv-2]). We conjecture that meromorphic mappings into spaces of class $\mathcal{G}_{k}$ are "almost Hartogs-extendable" in bidimension $(n, k)$ for all $n \geq 1$ :

Conjecture 1. Every meromorphic map $f: H_{n}^{k}(r) \rightarrow X$ with $X \in \mathcal{G}_{k}$ which is disk-convex in dimension $k$, extends to a meromorphic map from $\Delta^{n+k} \backslash A$ to $X$, where $A$ is an analytic subvariety of $\Delta^{n+k}$ (maybe empty) of pure codimension $k+1$. Moreover, if $A \neq \emptyset$, then for every sphere $\mathbb{S}^{2 k+1}$ embedded in $\Delta^{n+k} \backslash A$ in such a way that $\left[\mathbb{S}^{2 k+1}\right] \neq 0$ in $H_{2 k+1}\left(\Delta^{n+k} \backslash A, \mathbb{Z}\right)$, also $f\left(\mathbb{S}^{2 k+1}\right)$ is not homologous to zero in $X$.

In [Iv-2] this conjecture is proved for the case $k=1$.

ConjeCture 2. Let $M$ be a strictly pseudoconvex, compact contour of real dimension $2 k+1$ in a $k$-disk-convex complex manifold $X \in \mathcal{G}_{k}, k \geq 2$. Then the Plateau problem for $M$ has a solution iff $M$ is homologous to zero in $X$.

This conjecture would follow from the first one, but is probably easier.

Conjecture 3. Let $M$ be a strictly pseudoconvex, compact, three-dimensional contour in a compact manifold $X$. Then $M$ bounds an abstract Stein domain.

To our knowledge, this question is open even for $X=\mathbb{C P}^{3}$.

\section{References}

[Ba] D. Barlet, Espace analytique réduit des cycles analytiques complexes compacts d'un espace analytique complexe de dimension finie, in: Séminaire Norguet IX, Lecture Notes in Math. 482, Springer, 1975, 1-158.

[Db] P. Dolbeault, Geometric measure theory and the calculus of variations, in: Proc. Sympos. Pure Math. 44, Amer. Math. Soc., 1986, 171-205. 
[Db-H] P. Dolbeault et G. Henkin, Surfaces de Riemann de bord donné dans $\mathbb{C P}^{n}$, in: Contributions to Complex Analysis and Analytic Geometry, H. Skoda et al. (eds.), Aspects of Math. E26, Vieweg, 1994, 163-187.

[Ga] P. Gauduchon, Les métriques standard d'une surface à premier nombre de Betti pair, Astérisque 126 (1985), 129-135.

$[\mathrm{H}] \quad$ R. Harvey, Holomorphic chains and their boundaries, in: Proc. Sympos. Pure Math. 30, Part 1, Amer. Math. Soc., 1977, 307-382.

[H-L] R. Harvey and H. Lawson, An intrinsic characterization of Kähler manifolds, Invent. Math. 74 (1983), 169-198.

[H-L] G. Henkin and J. Leiterer, Theory of Functions on Complex Manifolds, Monographs in Math., Birkhäuser, 1984.

[Iv-1] S. Ivashkovich, The Hartogs-type extension theorem for the meromorphic maps into compact Kähler manifolds, Invent. Math. 109 (1992), 47-54.

[Iv-2] -, Continuity principle and extension properties of meromorphic mappings with values in non Kähler manifolds, MSRI Preprint No. 1997-033, xxx.math-archive: math.CV/9704219.

[Iv-3] - One example in concern with extension and separate analyticity properties of meromorphic mappings, xxx.math-archive: math.CV/9804009, to appear in Amer. J. Math.

[Ka-1] M. Kato, Examples on an extension problem of holomorphic maps and holomorphic 1-dimensional foliations, Tokyo J. Math. 13 (1990), 139-146.

[Ka-2] -, Compact quotient manifolds of domains in a complex 3-dimensional projective space and the Lebesgue measure of limit sets, ibid. 19 (1996), 99-119.

[Ka-3] -, Compact complex manifolds containing "global" spherical shells I, in: Proc. Internat. Sympos. Algebraic Geometry, Kyoto, 1977, 45-84.

[Kl] M. Klimek, Pluripotential Theory, London Math. Soc. Monographs (N.S.) 6, Cambridge Univ. Press, 1991.

[Lg] P. Lelong, Plurisubharmonic Functions and Positive Differential Forms, Gordon and Breach, New York, 1969.

[Lv] E. Levi, Studii sui punti singolari essenziali delle funzioni analitiche di due o più variabili complesse, Ann. Mat. Pura Appl. 17 (1910), 61-87.

[Re] R. Remmert, Holomorphe und meromorphe Abbildungen komplexer Räume, Math. Ann. 133 (1957), 328-370.

[Rs] H. Rossi, Attaching analytic spaces to an analytic space along a pseudoconvex boundary, in: Proc. Conf. Complex Analysis (Minneapolis, 1964), Springer, 1965, $242-256$.

Université de Lille-I

IAPMM

U.F.R. de Mathématiques

59655 Villeneuve d'Ascq Cedex, France

E-mail: ivachkov@gat.univ-lille1.fr
Academy of Sciences of Ukraine

Naukova 3/b

290053 Lviv, Ukraine 